\title{
Hepatitis B Virus Promotes Hepatocellular \\ Carcinoma Development by Activating GP73 to Repress the Innate Immune Response
}

Zhixin Liu ( Izx20022456@126.com )

Hubei University of Medicine

Long Liu

Hubei University of Medicine

Yanping Huang

Hubei University of Medicine

Yanan Fu

Hubei University of Medicine

Jingjing Rao

Hubei University of Medicine

Feng Zeng

Hubei University of Medicine

Manshan Ji

Hubei University of Medicine

Xiang Xu

Hubei University of Medicine

Jianyong Zhu

Hubei University of Medicine

Weixing Du

Hubei University of Medicine

\section{Research Article}

Keywords: Hepatitis B Virus, Hepatocellular Carcinoma, Golgi Protein 73

Posted Date: March 28th, 2022

DOl: https://doi.org/10.21203/rs.3.rs-1349279/v2

License: (1) (1) This work is licensed under a Creative Commons Attribution 4.0 International License.

Read Full License 


\section{Abstract}

\section{Background}

Hepatitis B virus (HBV) causes acute and chronic infection in the clinic. Hepatocellular carcinoma (HCC) is closely linked to HBV infection. Serum Golgi protein 73 (GP73) increases during HBV infection. However, the role of GP73 during HBV infection and the occurrence of HBV-related HCC is still poorly understood.

\section{Methods}

The underlying role of HBV-induced GP73 in regulating HCC development was investigated in this study. GP73 expression in HBV-related clinical HCC tissues and in HBV infected hepatoma cells and primary human hepatocytes were evaluated by immunohistochemistry, ELISA, Western Blotting and Quantitative real-time PCR (QRT-PCR) analysis. GP73 mediated tumorigenesis were detected by flow cytometry, QRTPCR, xenograft nude mice and sphere formation assay. The effects of GP73 and HBV infection on host's innate immune responses in hepatocytes were further investigated by Western Blotting and Quantitative real-time PCR (QRT-PCR) analysis.

\section{Results}

Initially, we discovered that HBV-positive HCC tissues had significantly higher expression of GP73. Ectopic expression of the HBV gene could induce GP73 expression in primary human hepatocytes and hepatoma cells in vitro. In addition, we discovered that GP73 promotes HCC in both normal liver cells and hepatoma cells. We also found that ectopic expression of HBV genes increases GP73 expression, suppressing the host's innate immune responses in hepatocytes.

\section{Conclusions}

Our results demonstrate that HBV facilitates HCC development by activating GP73 to repress the host's innate immune response. This study adds to our understanding of the pathogenesis of HBV infectioninduced HCC. The findings also provide preclinical support for GP73 as a potential HCC prevention or treatment target.

\section{Introduction}

Hepatitis B virus (HBV) causes acute and chronic infection in the clinic [1]. More than 2 billion people worldwide have been infected with the HBV [2]. About $5-10 \%$ of adults develop into chronic carriers after acute HBV infection, and up to $30 \%$ of chronic carriers eventually develop hepatitis, fibrosis, or cirrhosis, resulting in hepatocellular carcinoma (HCC) [3]. Chronic HBV infection can easily lead to the occurrence of HCC $[4 ; 5]$, but the molecular mechanism of HBV infection-induced HCC is still not fully understood. 
During HBV infection, hepatocytes begin to express some proteins abnormally, among which GP73 (Golgi protein 73) is one of the upregulated proteins [6]. GP73 is a Golgi protein identified by Kladney et al. [7] in a study of adult giant cell hepatitis (GCH). GP73 is rarely expressed in hepatocytes in normal liver tissues, mainly in bile duct epithelial cells in the portal area [7]. Clinical evidence has shown that the concentration of serum GP73 is significantly higher in patients with hepatocyte damage caused by viral invasion [8]. However, most studies focus on the relationship between abnormal expression of GP73 and liver carcinogenesis [9; 10; 11], and the role of GP73 during HBV infection is poorly understood. Here, we identified that GP73 promotes HCC development by repressing the host's innate immune response. Here, we found that HBV-positive HCC tissues had higher levels of GP73 than HBV-negative HCC tissues. The forced expression of HBV genes activated GP73 in primary human hepatocytes (PHHs) and hepatoma cells. More importantly, genetic overexpression of GP73 promoted the appearance and self-renewal in liver cancer stem cells (CSCs) and enhanced HepG2 and Huh7 cell tumorigenesis in nude mice. We further found that GP73 repressed the expression of NK-KB, IFN- $\lambda 1$, IFN- $\beta$, TNF- $\alpha$, and IL- 6 . These results indicated that the HBV facilitates HCC development by activating GP73 to repress the host's innate immune response.

\section{Materials And Methods}

\section{Clinical samples and immunohistochemistry}

Formalin-fixed and paraffin-embedded liver cancer tissues were obtained from patients undergoing surgery in Renmin Hospital, Hubei University of Medicine, ShiYan, China. Six HBsAg positive HCC tissues and six HBsAg negative HCC tissues were selected for analysis. The selected pathological tissues were negative for hepatitis A, C, D, and $E$ viruses and negative for human immunodeficiency virus (HIV). As previously described, immunohistochemical staining and Western blot analysis were performed [12]. The antibody used for staining was anti-GP73 (\#ab109628, Abcam). The study was conducted according to the principles of the Helsinki Declaration and approved by the Institutional Review Committee of Hubei Medical University according to its guidelines for protecting human subjects. Each participant received written informed consent.

\section{Xenograft nude mice}

Male BALB/CA-nu mice (19.3-23.6g) aged 5 weeks were purchased from Beijing HFK Bioscience Co., Ltd. (Beijing, China). The tumorigenicity assay in nude mice was performed as described previously [12]. Animal conservation and sacrifice are carried out according to the methods approved by the Animal Care and Use Committee of Animal Experimental Center of Hubei Medical University.

\section{Cell culture and transfection}

Primary human hepatocytes (PHHs) were purchased from the Research Institute for Liver Diseases (Shanghai, China) and cultured as described previously [13]. HepG2 cells, Huh-7 cells, HepG2.2.15 cells, and HEK293T cells were purchased from the China Center for Type Culture Collection (CCTCC, Wuhan, 
China). L02 cells and HepG2-NTCP cells were kindly provided by Dr. Jianguo Wu of Wuhan University, China. HepG2 cells, HepG2-NTCP cells, HepG2.2.15 cells, Huh-7 cells, L02 cells, and HEK293T cells were cultured as previously reported [14]. Lipofectamine ${ }^{\circledR} 3000$ (Thermo Fisher Scientific, USA) was employed to transfect cells with plasmid or small interfering RNAs according to the manufacturer's instructions.

\section{Plasmid construction}

Specific primers were used to amplify the coding region of GP73 by PCR (forward: 5'-GCG GAA TTC ATG ATG GGC TTG GGA AAC-3'; reverse: 5'-CCG CTC GAG TCA GAG TGT ATG ATT CCG-3'). The GP73 PCR product was inserted into the $\mathrm{pCMV}$-Tag2B vector.

For stably overexpressing cell line construction, the lentivirus plasmid pWPXLD (Addgene plasmid \# 12258) was modified by inserting a T2A peptide between the gene of interest and the GFP gene. Then, the GP73 gene was amplified by PCR and inserted into the modified lentiviral plasmid pWPXLD.

All constructs were confirmed by DNA sequencing.

\section{ELISA, Western Blotting and Immunofluorescence}

For ELISAs, cell culture supernatants were collected to detect the levels of HBeAg and HBsAg with an ELISA kit (Kehua Bio-Engineering, Shanghai, China). Serum GP73 was detected by an ELISA kit were purchased from Hotgen Biotech, China.

Western blot analysis was performed as described previously [12]. Antibodies were used to detect HA tags (\#SAB2702196, Sigma), FLAG tags (\#F7425, Sigma), GP73 (\#ab109628, Abcam), NF-kB (p50) (\#ab7549, Abcam), IFN- $\beta$ (\#ab180616, Abcam), IFN- $\lambda 1$ (\# MA5-30682, Invitrogen), IL-6 (\#ab9324, Abcam), TNF-a (\#ab6671, Abcam) and $\beta$-actin (\#A1978, Sigma). ImageJ (http://rsb.info.nih.gov/ij/) software was employed for band intensity quantification of the Western blot results.

\section{Quantitative RT-PCR analysis}

Total RNA of cells was extracted using TRIzol reagent (Invitrogen). RT-PCR primers as follows: GP73 forward: 5'-CAC AAG GGA AGG GAA ACG TG-3', GP73 reverse: 5'-CGA AGC CTC TTC CAC CTA CA-3'; KIf4 forward: 5'- ATT ACC CAT CCT TCC TGC CC -3', KIf4 reverse: 5'-CAC GAT CGT CTT CCC CTC TT-3'; Sox2 forward: 5'-AGC TCG CAG ACC TAC ATG AA-3', Sox2 reverse: 5'-TGG AGT GGG AGG AAG AGG TA-3'; Nanog forward: 5'-ACC CAG CTG TGT GTA CTC AA-3', Nanog reverse: 5'-CTG CGT CAC ACC ATT GCT AT3'; c-Myc forward: 5'-ATT CTC TGC TCT CCT CGA CG-3', c-Myc reverse: 5'-AGC CTG CCT CTT TTC CAC A3'; Oct4 forward: 5'-AG AAC ATG TGT AAG CTG CGG-3', Oct4 reverse: 5'-GGT TCG CTT TCT CTT TCG GG3'; IFN- $\beta$ forward: 5'-CTG CAA CCT TTC GAA GCC TT-3', IFN- $\beta$ reverse: 5'-AAG CCT CCC ATT CAA TTG CC3'; IFN- $\lambda 1$ forward: 5'-GCT GGT GAC TTT GGT GCT AG-3', IFN- $\lambda 1$ reverse: 5'-AAG ACA GGA GAG CTG CAA CT-3'; IL-6 forward: 5'-AGT CCT GAT CCA GTT CCT GC-3', IL-6 reverse: 5'-CTA CAT TTG CCG AAG AGC CC3'; TNF- $a$ forward: 5'-GTC AAC CTC CTC TCT GCC AT-3', TNF-a reverse: 5'-CCA AAG TAG ACC TGC CCA GA-3'; NF-KB forward: 5'-AAT GGT GGA GTC TGG GAA GG-3', NF-KB reverse: 5'-TCT GAC GTT TCC TCT GCA CT-3; GAPDH forward: 5'- GGA AGG TGA AGG TCG GAG TCA ACG G-3', GAPDH reverse: 5'- CTC GCT 
CCT GGA AGA TGG TGA TGG G-3'. Expression level data were normalized to the GAPDH expression level in each sample.

HBV DNA was detected by TaqMan real-time PCR using the following primers: 5'-AGA AAC AAC ACA TAG CGC CTC AT-3', 5'- TGC CCC ATG CTG TAG ATC TTG-3' and probe 5'-TGT GGG TCA CCA TAT TCT TGG G$3^{\prime}$.

\section{Virus production and transduction of cell lines}

For HBV infection of HepG2-NTCP, the culture supernatant of the HepG2.2.15 cell line was concentrated 100 -fold by ultracentrifugation. The concentrated HBV stock titer (genome equivalents $/ \mathrm{ml}, \mathrm{GEq} / \mathrm{ml}$ ) was assessed using QRT-PCR. The multiplicity of infection (MOI) was defined as the genome equivalents per cell (GEq/cell). HepG2-NTCP cells were infected, as described previously [14].

For stable cell line generation, lentiviral particles were produced and transfected as previously reported [12].

\section{Flow cytometry}

A CytoFlex (Beckman Coulter, USA) or MoFlo flow cytometer (Beckman Coulter, USA) was employed to analyze GFP signals or cell immunophenotyping. Single-cell suspensions were tested by cell immunophenotyping analysis after cells were stained with fluorescently labeled antibodies: CD90-APC (clone 5E10, eBioscience, 1:100), CD133-APC (clone AC133, Miltenyi Biotec, 1:100), CD117-APC (clone 104D2, eBioscience, 1:100). Flow cytometry data were analyzed using CytExpert software (Beckman Coulter, USA).

\section{Sphere formation assay}

Sphere formation assays were performed as previously reported [12]. HepG2-GFP cells and HepG2-GP73 cells were plated in ultra-low attachment 6-well plates (Corning Inc., Corning, NY, USA) at a density of 2000 cells/well. For Huh7-GFP and Huh7-GP73, a single cell was successively sorted into ultra-low attachment 96-well plates (Corning Inc., Corning, NY, USA) by a MoFlo flow cytometer (Beckman Coulter, USA).

\section{Statistical analysis}

All experiments were performed in triplicate. All data were recorded as the means \pm standard deviation (SD) unless otherwise stated. Prism 5 software (GraphPad Software) was used for a statistical test. $P<$ 0.05 was considered statistically significant.

\section{Results}

\section{HBV induces GP73 expression in clinical HCC tissues}


It has been reported that viral invasion can induce the expression of GP73 in hepatocytes [15]. The concentration of serum GP73 is significantly increased in patients with liver injury caused by virus infection [8]. Clinical carcinoma tissues were analyzed to validate the role of GP73 in HBV-related HCC. The immunohistochemistry analysis showed that the expression of GP73 was higher in HBV positive $\mathrm{HCC}$ tissues than in HBV negative HCC tissues (Fig. 1A\&B). These results suggest that HBV infection induces GP73 expression in HCC tissues.

\section{HBV induces the expression of GP73 in hepatoma cells and primary human hepatocytes}

The abnormal expression of GP73 is also closely related to HCC development (Liu et al. 2016). Huh7 cells and HepG2 cells were transfected with pHBV1.3 to identify if HBV infection promotes the expression of GP73. After 48 hours, the protein levels of HBeAg and HBsAg in cell culture supernatants were detected by ELISA. The results showed that the expression of HBeAg and HBsAg in cells was not affected (Fig. 2A). To further confirm whether HBV antigens activate GP73 expression, we analyzed the expression of GP73 by Western blot and real-time PCR analysis in PHHs genetically modified to overexpress pHBV1.3. When HBV genes were overexpressed in PHHs, GP73 expression increased (Fig. 2C, D\&E).

Moreover, we investigated whether GP73 expression is activated by HBV infection by imitating the natural infection of HBV in HepG2-NTCP cells. We first compared the HBV infection efficiency of HepG2-NTCP cells with that of HepG2 cells. When HepG2-NTCP cells and HepG2 cells were infected with HBV, HBeAg and HBsAg expressions were upregulated in HepG2-NTCP cells (Fig. 3A\&B). Further analysis of HBV coreassociated DNA and secreted HBV core-associated DNA confirmed that HBV replication proceeded in HepG2-NTCP cells (Fig. 3C). Subsequently, we sought to investigate whether HBV infection could affect GP73 expression in the HepG2-NTCP system. When analyzing GP73 expression in HBV infected HepG2NTCP cells, we found that the mRNA expression level of GP73 was upregulated (Fig. 3D).

Accordingly, the above results suggest that HBV induces the expression of GP73.

\section{GP73 activates CSC-related genes in L02 and HepG2 cells}

It has been reported that there is a close correlation between HCC and abnormal GP73 expression [6; 11]. However, direct evidence that GP73 induces hepatocarcinogenesis is still lacking. Here, we investigated the roles of GP73 in activating CSC-related genes in L02 cells and HepG2 cells. We generated 4 stable overexpression cell lines (L02-GFP, L02-GP73, HepG2-GFP, and HepG2-GP73) that stably express GFP and GP73 proteins. Then, the CSC surface markers (CD117, CD133, and CD90) of 4 stable cell lines were analyzed. The results have shown that the proportion of $\mathrm{CD}_{133^{+}}$cells increased from $0.39-4.81 \%$ in $\mathrm{LO} 2$ cells (Fig. 4A, left) and from 2.25-11.70\% in HepG2-GFP cells stably expressing GP73 (Fig. 4A, right). We also found that the CD177 ${ }^{+}$proportion increased in L02 cells (from 0.37 to $9.91 \%$, Fig. 4B, left) and HepG2 cells (from $2.46-11.84 \%$, Fig. 4B, right) stably expressing GP73. Similarly, flow cytometry revealed that the $\mathrm{CD}_{90} 0^{+}$population increased from 0.38 to $9.60 \%$ (Fig. 4C, right) in L02 cells and from 2.16- 
28.40\% (Fig. 4C, right) in HepG2 cells. QRT-PCR results showed that Sox2, KIf4, Nanog, c-Myc, and Oct4 were upregulated in HepG2 and L02 cells, which overexpressed GP73 (Fig. 4D). These results demonstrate that GP73 activates CSC-related genes in L02 and HepG2 cells.

\section{GP73 facilitates self-renewal and stemness in normal liver and hepatoma cells}

Because GP73 activates CSC-related genes in L02 and HepG2 cells, we further measured the sphere formation ability of HepG2-GP73 cells and Huh 7-GP73 cells and measured the migration ability of L02GP73 cells. Sphere formation assays revealed that the colony number of HepG2-GP73 cells was higher than control (Fig. 5A). In addition, the colony growth rate of Huh7-GP73 cells was faster than that of Huh7-GFP cells (Fig. 5B). These results suggest that GP73 enhances the colony-formation ability of HepG2 and Huh7 cells. Moreover, wound healing assays showed increased cell migration in L02-GP73 cells (Fig. 5C). The results above show that GP73 enhances self-renewal and stemness in normal liver and hepatoma cells.

\section{GP73 increases HepG2- and Huh7-mediated tumorigenesis in nude mice}

Because we found that the forced expression of GP73 enhanced self-renewal and stemness in Huh7 and HepG2 cells, the effect of GP73 on tumorigenesis in nude mice was further evaluated, and the results showed that tumors were induced in all nude mice injected with HepG2 and Huh7 cells (Fig. 6A). Tumour growth curves were drawn and showed that tumor volume increased over time (Fig. 6B). Furthermore, the average weight of the tumors was calculated. The average HepG2-GP73 and Huh7-GP73 tumor weights were significantly higher than the control tumors (Fig. 6C). These results indicate that GP73 enhances the tumorigenicity of HepG2 and Huh7 cells in nude mice.

\section{The HBV-induced expression of GP73 represses the expression of NF-KB, IFN- $\lambda 1$, IFN- $\beta$, TNF- $a$, and IL- 6}

The above results indicate that HBV activates GP73 expression to promote HCC development. We next sought to clarify the molecular mechanism by which GP73 facilitates HCC development. Previous reports have shown that host innate immunity is activated viral invasion and produces a series of antiviral cytokines [16]. We performed a series of experiments to assess whether the expression of GP73 regulates the host innate response. When analyzing GP73 and NF-KB in two hepatoma cell lines (HepG2 and Huh7) transfected with pHBV1.3, we found that GP73 mRNA levels positively correlated with pHBV1.3 expression, and NF-KB mRNA levels negatively correlated with pHBV1.3 expression (Fig. 7A). Further study also revealed that the forced expression of HBV genes significantly upregulated GP73, which downregulated the expression of IFN- $\lambda 1$, IFN- $\beta$, TNF- - , and IL- 6 in HepG 2 and Huh7 cells at both the mRNA and protein levels (Fig. 7B-D). 


\section{HBV facilitates HCC development by activating GP73 to repress the host innate immune response}

Altogether, these results suggest that HBV promotes GP73 overexpression in cells, inhibits NF-KB and its downstream signaling pathways, results in inhibition of cellular innate immune response. The inhibition of cellular innate immune response is conducive to HBV replication, leading to the loss of monitoring of abnormal hepatocytes, which eventually results in HBV-related hepatocarcinogenesis (Fig. 8).

\section{Discussion}

Chronic HBV carriers are more likely to develop HCC than non-carriers $[17 ; 18 ; 19]$. HBV persistently replicates in hepatocytes without obvious cell damage or death, suggesting noncytopathic virus [20]. However, HBV-related HCC develops in an inflammatory environment caused by chronic HBV infection, indicating that the occurrences of HCC are immune-mediated [21]. Continuous HBV replication in hepatocytes leads to persistent inflammation and prolonged CLD, the leading cause of HCC [22]. During HBV infection, hepatocytes begin to express certain proteins abnormally. The activation of certain proteins that attenuate host immune response after HBV infection may cause chronic infection.

Studies have shown that virus infection can activate the expression of GP73 in hepatocytes [8; 15]. We also found that HBV infection upregulates GP73 during hepatocarcinogenesis. The role of HBV in the activation of GP73 expression was further confirmed in HepG2-NTCP cells.

Previous reports have demonstrated that the concentration of serum GP73 is significantly upregulated in patients with CLD or HCC [11]. Although the relationship between clinical viral liver injury and abnormal expression of GP73 has been confirmed $[9 ; 10 ; 11]$, the role of GP73 in the development of HBV-related HCC is still poorly studied. We showed that GP73 promotes HCC development by repressing the innate immune response. Flow cytometry and QRT-PCR analysis revealed that GP73 overexpression upregulates the expression of CSC-related genes in L02 and HepG2 cells. Sphere formation assays showed that GP73 enhances the colony-formation ability of HepG2 and Huh7 cells. Wound healing assays revealed that GP73 facilitates the migration of L02 cells. More importantly, xenograft mouse analysis indicated that GP73 enhanced the tumorigenicity of HepG2 and Huh7 cells in nude mice.

For chronic HBV infection, the biggest challenge for viral survival in vivo is successfully escaping immune-mediated elimination. During chronic HBV infection, varying degrees of liver inflammation persists. Chronic HBV infection downregulates the expression of interferon, and interferon therapy shows a strong antiviral effect in HBV transgenic mice [23]. The imbalance of cytokine secretion in hepatocytes or leukocytes further hinders the immune system from clearing the virus. Moreover, the HBx antigen of HBV inhibits TNF-a- and FAS-mediated apoptosis by the activation of NF-KB [24]. The nuclear transcription factor NF-KB plays an important role in cellular innate immunity [25]. When potential pathogens invade a cell, NF-KB is activated and triggers the production of a series of antiviral cytokines (IFN- $\beta$, TNF- $\alpha$ ) [26]. In addition, NF-kB plays a key role in hepatocyte survival by intrahepatic balancing 
and inhibiting cytotoxic reactive oxygen species (ROS) accumulation [27]. Thus, NF-kB is essential for maintaining hepatocyte homeostasis [28].

This study evaluated the effect of HBV and GP73 on tumorigenicity and innate immune regulation. We have previously proved that forced expression of the HBV gene induces HCC development [12]. We found that GP73 also facilitates HCC development, and the HBV activated its expression. Further expression analysis showed that the expression of innate immune response-related factors (IFN- $\beta$, IFN- $\lambda 1$, IL- 6 , TNFa, and NF-KB) was inversely regulated by HBV in both HepG2 and Huh7 cells. Thus, our results indicate that HBV activates GP73 to inhibit cellular innate immunity, protecting HBV-infected cells from immunemediated cytotoxic lysis and facilitating HCC development.

\section{Conclusion}

In conclusion, our results reveal that HBV facilitates HCC development by activating GP73 to repress the host's innate immune response. Initially, HBV induces GP73 expression. Then, GP73 promotes HCC development by inhibiting the innate immune response through the NF-KB signaling pathway. Accordingly, HBV may co-opt GP73 to maintain a persistent infection by repressing cellular innate immunity through attenuating NF-KB activity, leading to the occurrence of HCC. This study provides a new perspective for exploring the pathogenesis of HBV-related HCC. The results also provide preclinical support for GP73 as potential HCC prevention or treatment targets.

\section{Declarations}

\section{Ethics approval and consent to participate}

This study was approved by the ethics committee of the Hubei University of Medicine.

\section{Consent for publication}

All authors agree with the publication of this paper in Infectious Agents and Cancer.

\section{Availability of data and materials}

All data and materials were presented in the text. It is also available from the corresponding author on request.

\section{Competing interests}

The authors declare that they have no competing interests.

\section{Funding}

This work was supported by research grants from the National Natural Science Foundation of China (81902066 and 82002149), Principle Investigator Program at Hubei University of Medicine 
(HBMUPI202102). The funder had no role in the study design, data collection and analysis, interpretation, and writing.

\section{Authors' contributions}

LL YPH and YNF performed the experiments and drafted the manuscript. JJR, ZF, MSJ and XX participated in the data analysis and specimen storage. JYZ, WXD and ZXL participated in the research design, literature review, and data examination. All authors read and approved the final manuscript.

\section{Acknowledgements}

Not applicable.

\section{References}

1. M.F. Yuen, D.S. Chen, G.M. Dusheiko, H.L.A. Janssen, D.T.Y. Lau, S.A. Locarnini, M.G. Peters, and C.L. Lai, Hepatitis B virus infection. Nature reviews. Disease primers 4 (2018) 18035.

2. A. Schweitzer, J. Horn, R.T. Mikolajczyk, G. Krause, and J.J. Ott, Estimations of worldwide prevalence of chronic hepatitis B virus infection: a systematic review of data published between 1965 and 2013. Lancet (London, England) 386 (2015) 1546-55.

3. C. Trépo, H.L. Chan, and A. Lok, Hepatitis B virus infection. Lancet (London, England) 384 (2014) 2053-63.

4. A. Arzumanyan, T. Friedman, I.O. Ng, M.M. Clayton, Z. Lian, and M.A. Feitelson, Does the hepatitis B antigen HBx promote the appearance of liver cancer stem cells? Cancer research 71 (2011) 3701-8.

5. A. Arzumanyan, H.M. Reis, and M.A. Feitelson, Pathogenic mechanisms in HBV-and HCV-associated hepatocellular carcinoma. Nature reviews. Cancer 13 (2013) 123-35.

6. W. Sai, L. Wang, W. Zheng, J. Yang, L. Pan, Y. Cai, L. Qiu, H. Zhang, W. Wu, and D. Yao, Abnormal Expression of Golgi Protein 73 in Clinical Values and Their Role in HBV-Related Hepatocellular Carcinoma Diagnosis and Prognosis. Hepatitis monthly 15 (2015) e32918.

7. R.D. Kladney, G.A. Bulla, L. Guo, A.L. Mason, A.E. Tollefson, D.J. Simon, Z. Koutoubi, and C.J. Fimmel, GP73, a novel Golgi-localized protein upregulated by viral infection. Gene 249 (2000) 53-65.

8. R.D. Kladney, X. Cui, G.A. Bulla, E.M. Brunt, and C.J. Fimmel, Expression of GP73, a resident Golgi membrane protein, in viral and nonviral liver disease. Hepatology (Baltimore, Md.) 35 (2002) 143140.

9. K. Jiang, W. Li, S. Shang, L. Sun, K. Guo, S. Zhang, and Y. Liu, Aberrant expression of Golgi protein 73 is indicative of a poor outcome in hepatocellular carcinoma. Oncology reports 35 (2016) 2141-50.

10. Y. Liu, X. Zhang, T. Sun, J. Jiang, Y. Li, M. Chen, Z. Wei, W. Jiang, and L. Zhou, Knockdown of Golgi phosphoprotein 2 inhibits hepatocellular carcinoma cell proliferation and motility. Oncotarget 7 (2016) 21404-15. 
11. Z. Zhang, Y. Zhang, Y. Wang, L. Xu, and W. Xu, Alpha-fetoprotein-L3 and Golgi protein 73 may serve as candidate biomarkers for diagnosing alpha-fetoprotein-negative hepatocellular carcinoma. OncoTargets and therapy 9 (2016) 123-9.

12. Z. Liu, X. Dai, T. Wang, C. Zhang, W. Zhang, W. Zhang, Q. Zhang, K. Wu, F. Liu, Y. Liu, and J. Wu, Hepatitis B virus PreS1 facilitates hepatocellular carcinoma development by promoting appearance and self-renewal of liver cancer stem cells. Cancer letters 400 (2017) 149-160.

13. X. Cao, Q. Ding, J. Lu, W. Tao, B. Huang, Y. Zhao, J. Niu, Y.J. Liu, and J. Zhong, MDA5 plays a critical role in interferon response during hepatitis $C$ virus infection. Journal of hepatology 62 (2015) 771-8.

14. J. Chen, W. Xu, Y. Chen, X. Xie, Y. Zhang, C. Ma, Q. Yang, Y. Han, C. Zhu, Y. Xiong, K. Wu, F. Liu, Y. Liu, and J. Wu, Matrix Metalloproteinase 9 Facilitates Hepatitis B Virus Replication through Binding with Type I Interferon (IFN) Receptor 1 To Repress IFN/JAK/STAT Signaling. Journal of virology 91 (2017).

15. L. Hu, W. Yao, F. Wang, X. Rong, and T. Peng, GP73 is upregulated by hepatitis $C$ virus (HCV) infection and enhances HCV secretion. PloS one 9 (2014) e90553.

16. J. Wu, and Z.J. Chen, Innate immune sensing and signaling of cytosolic nucleic acids. Annual review of immunology 32 (2014) 461-88.

17. R.P. Beasley, L.Y. Hwang, C.C. Lin, and C.S. Chien, Hepatocellular carcinoma and hepatitis B virus. A prospective study of 22707 men in Taiwan. Lancet (London, England) 2 (1981) 1129-33.

18. M. Sherman, Risk of hepatocellular carcinoma in hepatitis B and prevention through treatment. Cleveland Clinic journal of medicine 76 Suppl 3 (2009) S6-9.

19. V.T. Nguyen, M.G. Law, and G.J. Dore, Hepatitis B-related hepatocellular carcinoma: epidemiological characteristics and disease burden. Journal of viral hepatitis 16 (2009) 453-63.

20. M.A. Sells, M.L. Chen, and G. Acs, Production of hepatitis B virus particles in Hep G2 cells transfected with cloned hepatitis B virus DNA. Proceedings of the National Academy of Sciences of the United States of America 84 (1987) 1005-9.

21. B. Rehermann, and M. Nascimbeni, Immunology of hepatitis $B$ virus and hepatitis $C$ virus infection. Nature reviews. Immunology 5 (2005) 215-29.

22. G. Fattovich, T. Stroffolini, I. Zagni, and F. Donato, Hepatocellular carcinoma in cirrhosis: incidence and risk factors. Gastroenterology 127 (2004) S35-50.

23. S.M. Akbar, K. Inaba, and M. Onji, Upregulation of MHC class II antigen on dendritic cells from hepatitis $B$ virus transgenic mice by interferon-gamma: abrogation of immune response defect to a Tcell-dependent antigen. Immunology 87 (1996) 519-27.

24. J. Pan, Z. Lian, S. Wallet, and M.A. Feitelson, The hepatitis B $x$ antigen effector, URG7, blocks tumour necrosis factor alpha-mediated apoptosis by activation of phosphoinositol 3-kinase and betacatenin. The Journal of general virology 88 (2007) 3275-3285.

25. A. Dev, S. lyer, B. Razani, and G. Cheng, NF-KB and innate immunity. Current topics in microbiology and immunology 349 (2011) 115-43. 
26. S. Vallabhapurapu, and M. Karin, Regulation and function of NF-kappaB transcription factors in the immune system. Annual review of immunology 27 (2009) 693-733.

27. J.A. DiDonato, F. Mercurio, and M. Karin, NF-KB and the link between inflammation and cancer. Immunological reviews 246 (2012) 379-400.

28. T. Sakurai, S. Maeda, L. Chang, and M. Karin, Loss of hepatic NF-kappa B activity enhances chemical hepatocarcinogenesis through sustained c-Jun N-terminal kinase 1 activation. Proceedings of the National Academy of Sciences of the United States of America 103 (2006) 10544-51.

\section{Figures}

\section{Figure 1}

HBV infection facilitates GP73 expression in HCC tissues. (A) Immunohistochemical staining of HCC samples to detect GP73 is shown (scale bar $=500 \mu \mathrm{m}$ ). (B) Western blot analysis of HCC samples for the detection of GP73 is shown (left). Western blot band intensity was quantified using ImageJ software (right). Graphs show means $\pm \mathrm{SD}, \mathrm{n}=6 .{ }^{*} P<0.05$ compared with the control group.

\section{Figure 2}

HBV induces the activation of GP73. (A) pHBV1.3 at different doses were transfected into HepG2 and Huh7 cells. After 48 hours, the levels of HBsAg in cell culture supernatants were detected by ELISA. (B) pHBV1.3 at different doses were transfected into HepG2 and Huh7 cells. After 48 hours, the concentration of GP73 in whole-cell lysates was detected by ELISA. (C-E) The pHBV1.3 overexpression plasmid (0.4 $\mu \mathrm{g} / \mathrm{ml}$ ) was transfected into primary human hepatocytes (PHHs). The protein expression levels of $\mathrm{HBsAg}$ and GP73 were detected by Western blot (C). The mRNA expression levels of HBsAg and GP73 were analyzed by QRT-PCR (D \& E). Graphs show means $\pm \mathrm{SD}, \mathrm{n}=3 .{ }^{*} P<0.05,{ }^{*} P<0.01, * \star \star P<0.001$, compared with the control group.

\section{Figure 3}

\section{HBV induces the activation of GP73}

(A-D) HepG2 and HepG2-NTCP cells were infected with HBV at $1000 \mathrm{GE}$ per cell. After 48 hours, the levels of HBeAg (A) and HBsAg (B) in cell culture supernatants were detected by ELISA; HBV genomic DNA (C) levels in cells and cell culture supernatants were detected by QRT-PCR, and the mRNA expression level of 
GP73 (D) was detected by QRT-PCR. Graphs show means \pm SD, $\mathrm{n}=3 .{ }^{\star} P<0.05,{ }^{\star} P P<0.01$, $* \star \star P<0.001$, compared with the control group.

\section{Figure 4}

\section{GP73 activates CSC-related genes in L02 and HepG2 cells.}

(A-C) Percentage changes of $\mathrm{CD} 133^{+}(\mathrm{A}), \mathrm{CD}_{117^{+}}(\mathrm{B})$, and $\mathrm{CD} 90^{+}(\mathrm{C})$ cells in $\mathrm{LO2}$ and HepG2 cells were measured by flow cytometry after stable GP73 overexpression. L02-GFP and HepG2-GFP cells were employed as controls compared with L02-GP73 and HepG2-GP73 cells. (D-E) The mRNA levels of KIf4, Sox2, Nanog, c-Myc, and Oct4 in L02 cells (D) and HepG2 cells (E) were determined by QRT-PCR. Graphs show means $\pm \mathrm{SD}, \mathrm{n}=3 .{ }^{*} P<0.05,{ }^{\star *} P<0.01,{ }^{* \star *} P<0.001$, compared with the control group.

\section{Figure 5}

GP73 facilitates CSC generation in HepG2 and Huh7 cells. (A) HepG2-GFP and HepG2-GP73 cells were cultured in ultra-low attachment 6-well plates at 2000 cells/well density. Sphere formation assays of HepG2-GFP and HepG2-GP73 cells are shown. (B) Huh7-GFP and Huh7-GP73 cells were sorted into ultralow attachment 96 -well plates at a density of 1 cell/well by flow cytometry. The spheroid phenotype changes and numbers of HepG2-GFP and HepG2-GP73 cells were examined by microscopy. (C) A woundhealing assay to test the migration ability of L02-GP73 and L02-GFP cells is shown (Scale bar $=100 \mu \mathrm{m}$ ). Graphs show means $\pm \mathrm{SD}, \mathrm{n}=3 .{ }^{*} P<0.05,{ }^{*} P<0.01$, compared with the control group.

\section{Figure 6}

GP73 facilitates HepG2 and Huh7 tumorigenesis in nude mice. (A) The tumor images in each group are shown. (B) The average tumor volume in each group is shown. (C) The average tumor weight in each group is shown. Graphs show means $\pm \mathrm{SD}, \mathrm{n}=6 .{ }^{\star} P<0.05,{ }^{\star} P P<0.01$, compared with the control group.

\section{Figure 7}

\section{HBV induces GP73 expression to repress the expression of NF-KB, IFN- $\beta$, IFN- $\lambda 1$, IL-6, and TNF-a. (A-F)} Plasmid pHBV1.3 was transfected into HepG2 and Huh7 cells at different doses. After 48 hours, the expression levels of GP73, NF-KB, IFN- $\beta$, IFN- $\lambda 1$, IL-6, and TNF-a were detected by QRT-PCR (A-E) and 
Western blot $(\boldsymbol{F})$. Graphs show means $\pm \mathrm{SD}, \mathrm{n}=3 .{ }^{\star} P<0.05, * \star P<0.01$, ${ }^{\star \star \star} P<0.001$, compared with the control group.

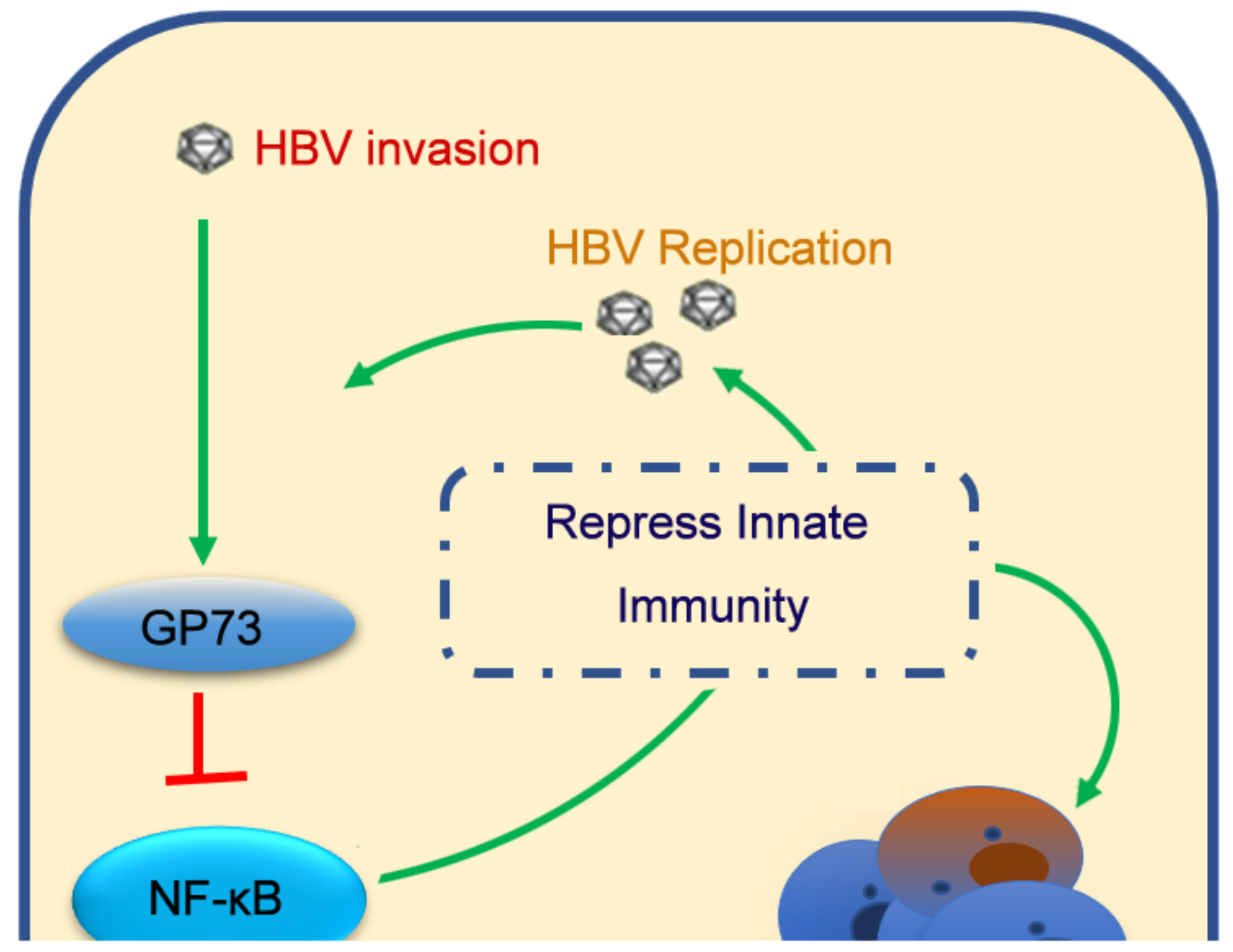

Figure 8

HBV facilitates HCC development by activating GP73 to repress the host's innate immune response. 
We propose that HBV promotes GP73 overexpression in cells, inhibits NF-KB and its downstream signaling pathways, and inhibits the cellular innate immune response. The inhibition of cellular innate immune response is conducive to HBV replication, leading to the loss of monitoring of abnormal hepatocytes, which eventually results in HBV-related hepatocarcinogenesis. 